\title{
Investigation of large-scale coherence in a turbulent boundary layer using two-point correlations
}

\author{
By B. GANAPATHISUBRAMANI, N. HUTCHINS, \\ W. T. HAMBLETON, E. K. LONGMIRE AND I. MARUSIC \\ Department of Aerospace Engineering and Mechanics, University of Minnesota, \\ 107 Akerman Hall, 110 Union Street SE, Minneapolis, MN 55455, USA \\ marusic@aem.umn.edu
}

(Received 24 February 2004 and in revised form 2 September 2004)

Stereoscopic particle image velocimetry (PIV) measurements are made in streamwisespanwise and inclined cross-stream planes (inclined at $45^{\circ}$ and $135^{\circ}$ to the principal flow direction) of a turbulent boundary layer at moderate Reynolds number $\left(R e_{\tau} \sim\right.$ 1100). Two-point spatial velocity correlations computed using the PIV data reveal results that are consistent with an earlier study in which packets of hairpin vortices were identified by a feature-detection algorithm in the log region, but not in the outer wake region. Both streamwise-streamwise $\left(R_{u u}\right)$ and streamwise-wall-normal $\left(R_{u w}\right)$ correlations are significant for streamwise displacements of more than 1500 wall units. Zero crossing data for the streamwise fluctuating component $u$ reveal that streamwise strips between zero crossings of 1500 wall units or longer occur more frequently for negative $u$ than positive $u$, suggesting that long streamwise correlations in $R_{u u}$ are dominated by slower streamwise structures. Additional analysis of $R_{w w}$ correlations suggests that the long streamwise slow-moving regions contain discrete zones of strong upwash over extended streamwise distances, as might occur within packets of angled hairpin vortices. At a wall-normal location outside of the log region $(z / \delta=0.5)$, the correlations are shorter in the streamwise direction and broader in the spanwise direction. Correlations in the inclined cross-stream plane data reveal good agreement with the streamwise-spanwise plane. $R_{u u}$ in the $45^{\circ}$ plane is more elongated along the in-plane wall-normal direction than in the $135^{\circ}$ plane, which is consistent with the presence of hairpin packets with a low-speed region lifting away from the wall.

\section{Introduction}

The year 2004 marks the centenary of Prandtl's original paper on boundary layers (Prandtl 1904). Even so, the study of turbulent boundary layers remains an active area of research. The majority of wall-bounded fluid flows, whether encountered in day-today life or engineering applications, are turbulent. A turbulent flow is characterized by three-dimensional chaotic motions often caused by coherent eddies of different sizes and orientation. A central goal has been to understand the physics and basic dynamics that govern these eddies, and hence develop better control and prediction strategies. Previous experimental and computational studies of turbulent boundary layers have revealed that eddy structures play a dominant role in turbulence production, and are major contributors to skin friction. In the case of a large airliner, up to $50 \%$ of the 
power expended by the engines is used to overcome turbulent skin friction drag. Hence, it is of primary importance to characterize the eddy structures, in particular the manner in which they evolve, regenerate, sustain turbulence and lead to increased skin friction drag.

The early work on structure identification in boundary layers goes back to flow visualizations first reported in the 1950s. Elongated streaky structures were observed in the near-wall region of a turbulent boundary layer by Hama, Long \& Hegarty (1957). A series of studies followed that concentrated largely on the near-wall regions (for example, Kline et al. 1967; Corino \& Brodkey 1967) and described regions of 'sweeps' ( $u>0, w<0, \mathrm{Q} 4$ events) and 'ejections' $(u<0, w>0$, Q2 events) that were considered the major source of turbulence production. Throughout this paper, $u, v$ and $w$ will be used to denote the fluctuating velocity components in the streamwise $x$, spanwise $y$ and wall-normal $z$ directions, respectively. Some workers (see references in Panton 1997) have proposed a model based on the existence of quasi-streamwise vortices in the near-wall region. In the present study, the focus is on the outer region (log region and beyond) of a turbulent boundary layer which is, in general, believed to be populated by larger structures that have grown beyond the near-wall regime of quasi-streamwise vortices.

There are several schools of thought regarding the structure of a turbulent boundary layer in the outer region. For example, Adrian, Meinhart \& Tomkins (2000) and Ganapathisubramani, Longmire \& Marusic (2003) found evidence for the existence of 'hairpin packets', whereas Na, Hanratty \& Liu (2001) classified large-scale lowmomentum regions that produce Reynolds stress as 'superbursts'. In this paper, we examine statistical evidence from stereo PIV datasets to determine the dominant structure characteristics in the outer region. As will be shown, the resulting data are consistent with the presence of hairpin packets that envelope large-scale lowmomentum regions.

The rest of this section consists of a brief review of the relevant literature describing advances in the understanding of hairpin-shaped structures and their dynamics, followed by the objectives of this study. The brief review is classified into two categories, namely flow visualization and measurement where the instantaneous variation in velocity over a spatial region is examined, and statistical analysis where significant features over a large dataset are isolated and analysed using ensemble-averaging techniques.

\subsection{Flow visualization and measurement}

The existence of a hairpin-shaped vortical structure responsible for turbulence transport was first proposed by Theodorsen (1952). Offen \& Kline (1975) invoked a model in which hairpin-shaped structures are inclined at an angle with the wall, to explain the ejections (slow-moving fluid pushed away from the wall) and sweeps (high-speed fluid pushed towards the wall). Such events are evidently strong contributors to the Reynolds shear stress within the boundary layer and consequently to surface drag.

The presence of inclined hairpin vortices in the log and outer wake regions of turbulent boundary layers has also been evidenced in experiments. Bandyopadhyay (1980) and Head \& Bandyopadhyay (1981) performed visualizations in smoke-filled zero-pressure-gradient boundary layers, illuminated with a laser sheet inclined at angles of $45^{\circ}$ and $135^{\circ}$ with the principal flow axis. The data in $45^{\circ}$ planes showed clear elongated features while the $135^{\circ}$ planes exhibited mainly counter-rotating vortex cores adjacent to one another (in the spanwise direction). In addition, the authors 
visualized the streamwise-wall-normal plane, noting that multiple hairpin structures of different scales could occasionally stack together and convect as a coherent group.

Flow-visualization experiments by Acarlar \& Smith $(1987 a, b)$, theoretical studies by Smith et al. (1991), and the study of turbulent channel flow DNS datasets by Zhou et al. (1999) at low Reynolds number $\left(R e_{\tau}=U_{\tau} \delta / \nu=180\right.$, where $U_{\tau}$ is the skin friction velocity, $\delta$ is the boundary-layer thickness, in the case of a channel equal to the half-width, and $v$ is the kinematic viscosity) have demonstrated that, when a single hairpin vortex of sufficient circulation is inserted into a mean shear flow (in this case a channel flow), it could spawn a series of smaller hairpin vortices, and that these vortices would travel together in a group with a common convection velocity.

Adrian et al. (2000) performed particle image velocimetry (PIV) measurements in streamwise-wall-normal planes $((x, z)$-planes $)$ in a zero-pressure-gradient turbulent boundary layer across the Reynolds number range, $355<R e_{\tau}<2000$. The authors identified numerous hairpin vortex heads in the logarithmic and outer layers. They also observed many instances where groups of 5-10 vortex heads covering a spatial streamwise distance of $2 \delta$ convected with a uniform velocity (also noted by Head $\&$ Bandyopadhyay 1981). They also noted that the presence of these vortex heads could explain the multiple ejections and sweeps found in previous hot-wire measurements (Bogard \& Tiederman 1986, Tardu 1995). The authors termed such a group of hairpin vortices a 'hairpin packet'. The existence of vortices organized within packets can also help explain the long tails in streamwise velocity autocorrelations within the boundary layer as observed by various workers (Townsend 1976; Brown \& Thomas 1977; Kovasznay, Kibens \& Blackwelder 1970). Adrian et al. (2000) also proposed the existence of a nested arrangement of hairpin packets across the boundary layer. They postulated that packets of larger hairpin vortices in the outer wake region would skip over packets of smaller hairpin vortices in the log region. The streamwise separation between individual hairpins in the outer wake region would be greater than the separation in the log region.

Ganapathisubramani et al. (2003) used a feature-detection algorithm to identify and examine vortical structures and packets occurring in individual velocity fields obtained in streamwise-spanwise planes using stereoscopic particle image velocimetry (PIV). The algorithm was designed such that, it searched for regions of strong positive and negative wall-normal vorticity $\left(\omega_{z}\right)$. Further, it looked for points of strong instantaneous Reynolds shear stress $(-u w)$ between the region of positive and negative vorticity. It then used the identified points as seed points for a region growing algorithm to isolate a region of low momentum (or slow-moving fluid) enveloped by cores of vortices that produce strong Reynolds shear stress. Such a region identified by the algorithm was labelled a 'hairpin packet' and its primary features, such as length, width, swirl strength of cores and contribution to total Reynolds shear stress were recorded. The algorithm identified many elongated packets within the log region $\left(z^{+}=92\right.$ and 150$)$, but few in the outer region $(z / \delta=0.2$ and 0.5$)$. The packets typically had vortices with two legs (counter-rotating) on either side of the low-speed region. Sometimes legs were offset in the streamwise direction. Not all vortices had two legs, implying the existence of asymmetric (cane-type) structures as well. Although the packets identified in the log layer occupied only $4-5 \%$ of the total streamwisespanwise area examined, they were found to contribute $25-28 \%$ of the total Reynolds shear stress $-\overline{u w}$. This estimate is conservative as it considers the contribution from the identified low-speed regions only and does not account for the associated Q4 contributions from either side of this region. 
An alternative view on the structure of a turbulent boundary layer uses the concept of a 'superburst' ( $\mathrm{Na}$ et al. 2001). In this model, large-scale Reynolds-stress-producing events (such as those documented by Kovasznay et al. 1970) are visualized as plumes of low-momentum fluid ejected outward from the wall (see Nagakawa, Na \& Hanratty 2003; Nagakawa \& Hanratty 2001). However, as discussed earlier, Adrian et al. (2000) and Ganapathisubramani et al. (2003) have shown that these large low-momentum regions, classified as 'superbursts' can occur within 'hairpin packets'.

\subsection{Statistical analysis}

Various workers have performed extensive two-point correlation studies based on hot-wire data using Taylor's hypothesis. Favre, Gaviglio \& Dumas (1957) acquired data at two wall-normal locations simultaneously in a turbulent boundary layer, and computed the transverse space-time double correlation (spanwise in space, streamwise in time). The measurements suggested that the eddy structure had an oblique pattern where the outer parts (farther from the wall) were further downstream than those closer to the wall. Kovasznay et al. (1970) constructed contour plots of space-time correlation of the three velocity components from fixed-probe data at a wall-normal location of $z / \delta=0.5$. They concluded that the individual 'bulges' or 'bursts' are strongly three-dimensional. They also found that these events are elongated in the streamwise direction and comparatively compact in the spanwise direction. Tritton (1967) found a decreasing trend in the streamwise length with wall-normal distance. Krogstad \& Antonia (1994) found long tails (extending beyond $2 \delta$ ) in the streamwise velocity autocorrelation in the streamwise direction of a turbulent boundary layer. The spanwise length scales were smaller (less than $0.5 \delta$ ). However, the spanwise velocity correlations were short (less than $0.5 \delta$ ) in both streamwise and spanwise directions. Based on simulation data, Moin \& Kim (1985) concluded that the two-point correlations strongly support a flow model with hairpin vortices inclined at $45^{\circ}$ to the wall in a channel flow. The authors also found that the size of vortices increased with wallnormal location.

Willmarth \& Lu (1972) and Blackwelder \& Kaplan (1976) performed a detailed study using conditional and phase averaging techniques to obtain an 'average' structure. They conditionally averaged on sweep and ejection events and concluded that sweeps and ejections of certain sizes make an appreciable contribution to the Reynolds shear stress and therefore to the drag on the surface.

Townsend (1976) showed that a pair of roller eddies inclined at an angle of $30^{\circ}$ is consistent with the two-point correlation measurements performed by Grant (1958). Townsend also claimed that 'attached' double-roller eddies are the dominant structures in the turbulent boundary layer. Perry \& Chong (1982) formulated a model based on Townsend's attached eddy hypothesis, featuring ' $\Lambda$ ' shaped vortices of different scales inclined at a fixed angle. Later, Perry \& Marusic (1995) and Marusic \& Perry (1995) refined this model to compute second-order statistics and found good quantitative agreement with experimental results. However, the agreement in velocity statistics using representative (average) ' $\Lambda$ ' shaped vortices does not necessarily imply that individual instantaneous hairpin vortices are always symmetric. The existence of hairpin structures of various shapes and sizes including asymmetric hairpins in the region above the buffer layer were documented by Robinson (1991) in his analysis of the early low-Reynolds-number $\left(R e_{\tau}=300\right)$ DNS dataset generated by Spalart (1988).

Christensen \& Adrian (2001) used linear stochastic estimation on PIV data to compute the conditionally averaged velocity field associated with swirling motion. The PIV 
data was in $(x, y)$-planes, from turbulent channel flow at $R e_{\tau}=547$ and 1734 . They found that the conditional structure consisted of a series of hairpin vortex heads along a line inclined at $12^{\circ}-13^{\circ}$ with the wall. This result is consistent with previous observations relating to hairpin packets. Marusic (2001) refined a previous model based on the attached eddy hypothesis to include vortex packets and found that packets of eddies could match the turbulence statistics in the logarithmic region better, in particular the long tails found in streamwise velocity fluctuation autocorrelations.

Tomkins \& Adrian (2003) studied the spanwise growth of structures in a turbulent boundary layer based on PIV measurements in $(x, y)$-planes from the buffer layer to the top of the $\log$ layer at $R e_{\tau}=426$ and 2216. They found that the dominant motions are large-scale low-speed regions elongated in the streamwise direction. These regions are bounded by an arrangement of vortices organized in the streamwise direction similar to those found by Ganapathisubramani et al. (2003). The authors found that the spanwise length scale based on two-point correlations varies linearly with distance from the wall, revealing a self-similar growth of an average spanwise structure. However, examination of instantaneous velocity fields indicated no obvious growth of individual structures. They proposed that this scale growth occurs by merging of vortex packets on an eddy-by-eddy basis through a vortex reconnection mechanism.

\subsection{Objectives}

The objective of this work is to present ensemble-averaged statistics based on the instantaneous velocity fields described in Ganapathisubramani et al. (2003), to provide additional evidence concerning large-scale spatially coherent features in the flow. The results presented are based on stereoscopic PIV datasets in the streamwise-spanwise planes $(x-y)$ of a turbulent boundary layer $\left(R e_{\tau} \sim 1100\right)$ in the logarithmic region (taken here nominally as $z^{+}=z U_{\tau} / v>70$ and $z / \delta<0.2$ ) and beyond. Explicit twopoint spatial correlations of all three velocity fluctuations in $(x, y)$-planes are presented. The results also include a detailed comparison with two-point correlations obtained from PIV data in planes inclined at $45^{\circ}$ and $135^{\circ}$ to the streamwise-spanwise planes. The motivation for this comparison study is to provide a comprehensive view of the true three-dimensional spatial nature of velocity correlations, without using Taylor's hypothesis.

\section{Facility and methods}

\subsection{Experimental set-up}

The experiments were performed in a suction-type boundary-layer wind tunnel shown in figure 1(a) with a variable-pitch blade fan driven by a $40 \mathrm{hp}$ motor. The speed of the tunnel is controlled manually, by adjusting the pitch of the blades and by a Toshiba $\mathrm{H} 3$ variable-torque adjustable-speed drive. The flow conditioning includes a hexagonal honeycomb cell flow straightener and three stainless steel screens. The contraction is three-dimensional with a 9:1 area ratio. The working section is $1.22 \mathrm{~m}$ wide, $4.75 \mathrm{~m}$ long and $0.3 \mathrm{~m}$ high. The tunnel floor is made of $19.05 \mathrm{~mm}$ aluminium tooling plates, except in the region of measurement planes. In the measurement region, a glass section $(0.5 \mathrm{~m}$ long and $1 \mathrm{~m}$ wide) provides optical access for PIV measurements, and the sidewalls are fitted with glass windows. Otherwise, the sidewalls are constructed of Plexiglas. The boundary layer was tripped with a cylindrical trip wire $1.5 \mathrm{~mm}$ in diameter, placed at the beginning of the working section. Measurement planes were located $3.3 \mathrm{~m}$ downstream of a trip wire in a zero-pressure-gradient flow. The freestream velocity $U_{\infty}$ was $5.9 \mathrm{~m} \mathrm{~s}^{-1}$, and hot-wire measurements indicated that the 

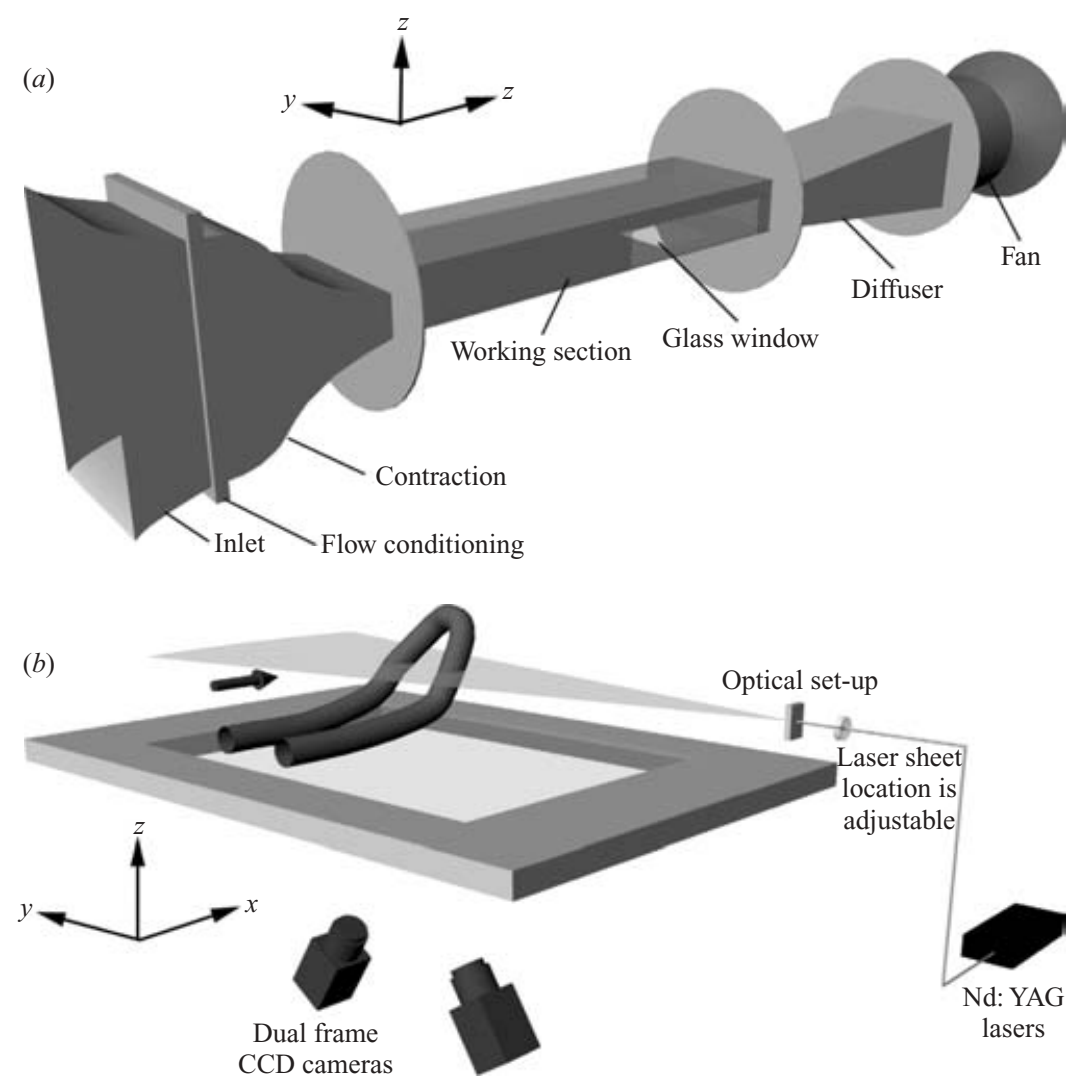

FIGURE 1. (a) Wind tunnel. (b) Stereoscopic PIV set-up.

freestream turbulence intensity was less than $0.2 \%$. The skin friction velocity $\left(U_{\tau}=\right.$ $0.244 \mathrm{~m} \mathrm{~s}^{-1}$ ), was estimated from the Clauser chart method, using a mean velocity profile measured using hot-wire anemometry. Throughout this paper, quantities nondimensionalized with $U_{\tau}$ and $v$ are denoted with a superscript + . The Reynolds number of the flow based on skin friction velocity and boundary-layer thickness was $R e_{\tau}=1060$. The boundary-layer thickness $(\delta)$ at the measurement location was $69 \mathrm{~mm}$.

The flow was seeded with olive oil droplets of size $\sim 1 \mu \mathrm{m}$. The olive oil droplets were generated using Laskin nozzles (see Raffel, Willert \& Kompenhans 1998, p. 22) and released just upstream of the tunnel inlet. Two pulsed Nd-YAG lasers (Big Sky CFR200, $120 \mathrm{~mJ}$ ) were used to illuminate a streamwise-spanwise plane of the boundary layer. A spherical bi-convex lens of focal length $1 \mathrm{~m}$ and a plano-concave cylindrical lens of focal length $-25 \mathrm{~mm}$ were used to fan the coincident beams into a sheet. The sheets were directed through one side window and oriented parallel with the bottom wall of the tunnel, as shown schematically in figure 1(b). Digital images were captured by TSI Powerview $2048 \times 2048$ pixel resolution cameras at $z^{+}=92$ and 150 , and by two Kodak Megaplus CCD cameras $(1024 \times 1024$ pixels $)$ at $z / \delta=0.2$ $\left(z^{+}=198\right)$ and $z / \delta=0.5\left(z^{+}=530\right)$. Nikon Micro Nikkor $60 \mathrm{~mm} \mathrm{f} / 2.8$ lenses were used with both camera types. The cameras had a stereoscopic angle of $15^{\circ}$ with respect to the perpendicular from the centre of the laser sheet, as shown in figure $1(b)$. A TSI synchronizer box controlled the firing of the lasers and the timing of the cameras. For all derived quantities, the interrogation spot size used was $16 \times 16$ pixels 
$(\sim 20 \times 20$ wall units) with $50 \%$ overlap. Hence, the spacing between adjacent vectors in either direction is 10 wall units $(\sim 0.65 \mathrm{~mm})$. An adaptive interrogation technique, where vector fields were computed in a coarse grid $(32 \times 32$ pixels $)$ and subsequently refined to a fine grid $(16 \times 16)$, was used. The vector field computation had two main sources of uncertainty. First, the Gaussian peak fit in the cross-correlation algorithm generated an uncertainty of approximately 0.1 pixels or $0.016 \bar{U}$ (where $\bar{U}$ is the mean velocity in the streamwise direction). Secondly, a residual error arose owing to the least-squares curve-fit in solving the four pixel-displacement equations in three unknowns (see Ganapathisubramani et al. 2002 for details). This residual error can play a major role in the uncertainty if concentration gradients in seeding occur within the flow field. In this experiment, such gradients were not significant, and this error was on average about half of the Gaussian error. The vector fields from the images acquired with the Megaplus cameras covered an area of $1.2 \delta \times 1.2 \delta$. For the Powerview cameras, the field size was approximately $2.4 \delta \times 2.4 \delta$ making the spacing between vectors similar to that in the other planes.

The values of $\bar{U}, \overline{u^{2}}, \overline{v^{2}}, \overline{w^{2}}$ and $-\overline{u w}$ computed from the PIV data agree well with previous hot-wire measurements (See Ganapathisubramani et al. 2003).

\subsection{Two-point correlations in streamwise-spanwise planes}

The instantaneous velocity realizations indicate the presence of hairpin structures and packets producing considerable Reynolds shear stress as a common and recurrent feature in the log-layer. This is well documented in Ganapathisubramani et al. (2003). However, it is useful to study the mean behaviour of these structures statistically. The typical organization of long low-speed zones enveloped by high-speed regions, along with associated Reynolds shear stress (indicative of intermittent wall-normal ejections over a packet) should become evident in the statistical analysis.

To quantify spatial coherence and understand the statistical significance of various features within the velocity fields, two-point correlations of the fluctuating velocity components were calculated. An overview of the notation used to describe two-point correlations follows. The two-point correlation coefficient between any two quantities $R_{A B}$ is defined as,

$$
R_{A B}=\frac{\overline{A(x, y) B(x+\Delta x, y+\Delta y)}}{\sigma_{A} \sigma_{B}},
$$

where, $\sigma_{A}$ and $\sigma_{B}$ are the standard deviations of $A$ and $B$, respectively, and $\Delta x$ and $\Delta y$ are the in-plane separations between the two components. The overline notation represents an ensemble average over multiple realizations.

All two-point correlation functions computed in the streamwise-spanwise planes were computed using fast Fourier transforms (FFT's). FFT libraries from FFTW (see website, http://www.fftw.org) were used to compute the transforms. Each vector field was zero padded to twice its original size (in both $x$ and $y$ directions), and a two-dimensional FFT operation was performed on both variables $A$ and $B$. The Fourier transforms of $A$ and $B$ are denoted by $\widehat{A}$ and $\widehat{B}$, respectively. The complex conjugate of $\widehat{A}$ is multiplied by $\widehat{B}$ to obtain the cross-power spectrum $S_{A B}$ which is ensemble averaged over all independent velocity field realizations.The inverse FFT of the ensemble-averaged cross-power spectrum gives the two-dimensional two-point correlation function $R_{A B}$. Since we have limited field of view, the number of data points over which the correlation is performed reduces linearly with the distance away from the origin in both directions. The decreasing sample size away from the origin was accounted for, and the correlations were normalized such that $R_{A A}(0,0)=1$. 

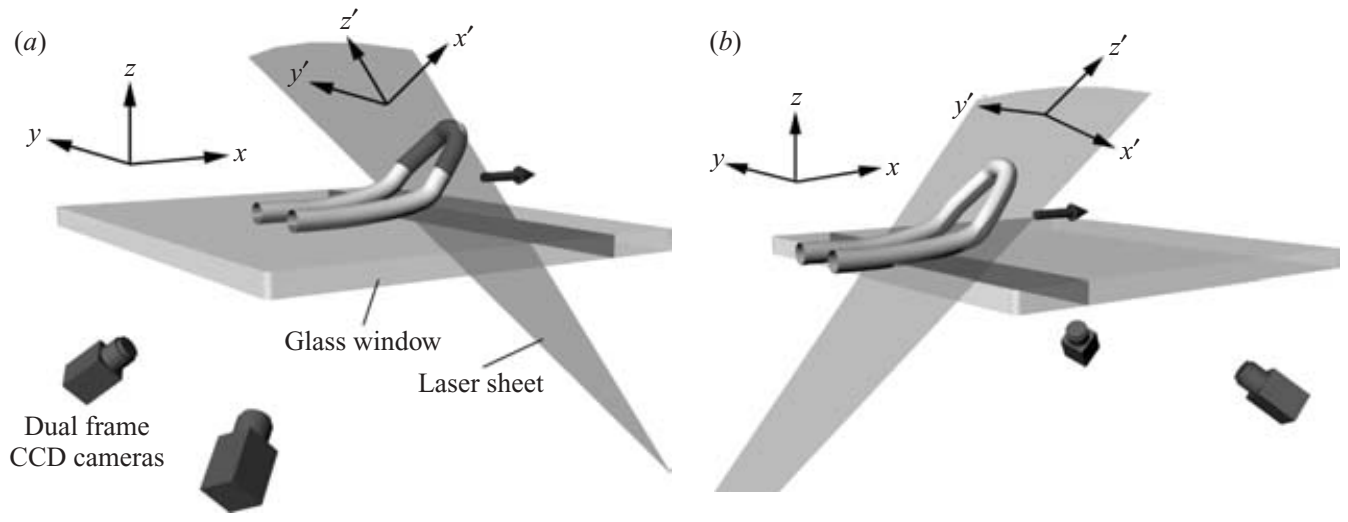

FIGURE 2. Inclined plane stereo PIV set-up, (a) $135^{\circ}$ case, (b) $45^{\circ}$ case.

The accuracy of the FFT routine was verified by comparing with an appropriately normalized direct correlation computed over real space using a smaller number of independent realizations. The use of FFTs was shown to have no adverse effect in the computed correlations. Thus, owing to significant computational efficiency, the FFT method was preferred over the direct method.

Two-point correlations of the fluctuating velocity components were computed using 750 independent velocity fields at each of the four wall-normal locations, $z^{+}=92$ and 150 in the $\log$ region and $z / \delta=0.2$ and $0.5\left(z^{+}=198\right.$ and 530, respectively) in the wake region.

\subsection{Inclined cross-stream planes}

Stereoscopic PIV data were also obtained in cross-stream planes inclined at $45^{\circ}$ and $135^{\circ}$ to the streamwise direction (figure 2). The experimental equipment used was the same as that described for the streamwise-spanwise plane experiments. Measurements were obtained in the same wind tunnel at a comparable Reynolds number, $R e_{\tau}=1142$ $\left(U_{\tau}=0.208 \mathrm{~m} \mathrm{~s}^{-1}, U_{\infty}=5.3 \mathrm{~m} \mathrm{~s}^{-1}\right.$ and $R e_{\tau}$ are within $\left.10 \%\right)$. An adaptive interrogation technique was used, where vector fields were computed in a coarse grid $(64 \times 64$ pixels $)$ and subsequently refined to a fine grid $(32 \times 32)$. The interrogation window size of $32 \times 32$ pixels corresponds to an area of $36 \times 36$ wall units. However, $50 \%$ overlap of the interrogation windows improved the vector resolution to $18 \times 18$ wall units. Smaller interrogation window sizes produced more missing vectors owing to large out-of-plane displacements. The Gaussian peak fit uncertainty was approximately 0.1 pixels $(0.015-0.033 \bar{U}$, depending on wall-normal position) and the average residual error was 0.1 pixels. The vector fields had a size of $1.8 \delta \times 1.5 \delta$ (width $\times$ height). Further experimental and parametric details on the inclined plane datasets are presented in Hutchins, Hambleton \& Marusic (2004). The transformed coordinate system for the inclined planes is shown in figure 2. In both cases, $x^{\prime}$ is out-of-plane, $z^{\prime}$ is the inplane component of the wall-normal coordinate and $y^{\prime}=y$ is the in-plane spanwise coordinate.

\subsection{Two-point correlations - inclined-planes}

Two-point correlation functions were computed for the inclined datasets in a box of width 900 wall units $(0.77 \delta)$ and height 1750 wall units (1.58) directly, using 500 independent realizations for the $45^{\circ}$ and $135^{\circ}$ cases. Since the vector fields are inhomogeneous in the wall-normal direction (owing to the presence of mean shear), 

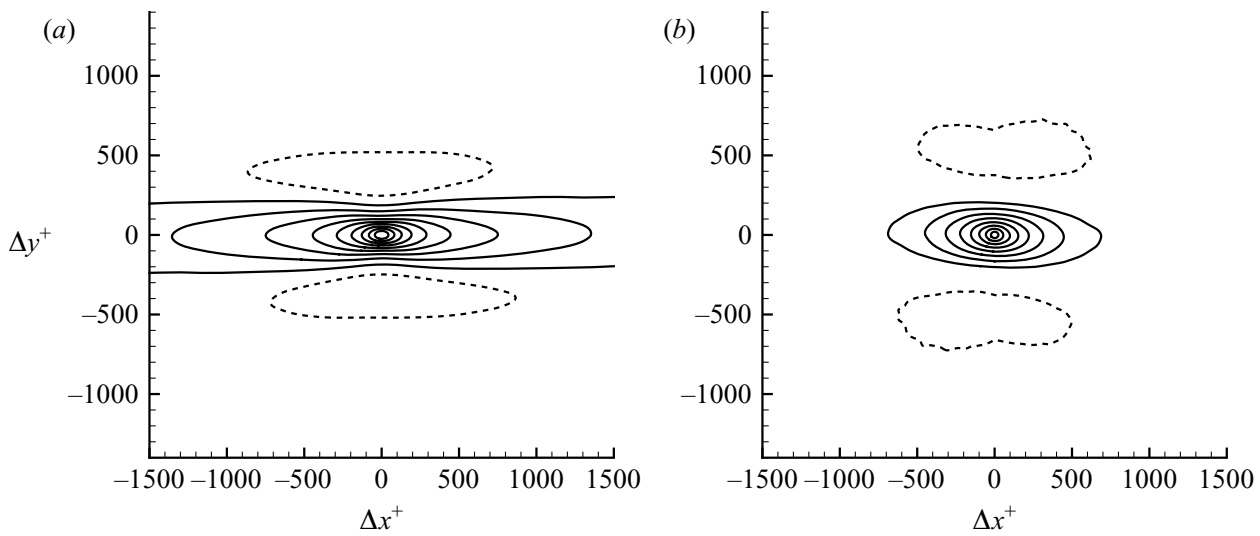

Figure 3. (a) $R_{u u}$ correlation at $z^{+}=92,(b) R_{u u}$ correlation at $z / \delta=0.5$. The contour levels for $R_{u u}$ range from -0.1 to 1.0 with spacing of 0.1 . Zero contours are not shown.

cross-correlations cannot be directly computed on the whole field. A unique twodimensional two-point correlation map of the form in (2.2) was constructed directly (in real-space) across a range of wall-normal heights (similar to Christensen \& Adrian 2001).

$$
R_{A B}\left(z_{r e f}\right)=\frac{\overline{A\left(y, z_{r e f}\right) B(y+\Delta y, z)}}{\sigma_{A}\left(z_{r e f}\right) \sigma_{B}(z)},
$$

where, $z_{\text {ref }}$ is the reference wall-normal location at which the correlation is computed. $\Delta y$ is the in-plane spanwise separation between $A$ and $B . \sigma_{A}\left(z_{r e f}\right)$ is the standard deviation of $A$ at $z_{\text {ref }}$ and $\sigma_{B}(z)$ is the standard deviation of $\mathrm{B}$ at location $z$.

All two-point correlations are computed in the inclined $\left(y^{\prime}, z^{\prime}\right)$-planes. Hence, a complete two-dimensional two-point cross-correlation function can be obtained at every $z^{\prime}$ location as a reference. In order to compare the inclined plane data with the streamwise-spanwise planes at a given wall-normal location $z$, the corresponding wall-normal reference location on the inclined plane $\left(z^{\prime}\right)$ is found by using simple trigonometric transformation $\left(z^{\prime}=\sqrt{2} z\right)$.

\section{Results and discussion}

\subsection{Streamwise-spanwise correlations}

Note that for all streamwise-spanwise correlation plots, the flow is from left to right. A positive $\Delta x$ corresponds to correlation $\left(R_{A B}\right)$ of variable $B$ at a downstream location from variable $A$ and a negative $\Delta x$ characterizes an upstream location of $B$ with respect to $A$. A similar sign convention applies to the spanwise direction.

Figures $3(a)$ and $3(b)$ show the two-point autocorrelation $R_{u u}$ at $z^{+}=92$ and $z / \delta=0.5$, respectively. For $z^{+}=92$, it is immediately obvious that there is significant spatial coherence in the streamwise direction, such that the correlation coefficient extends further than 1500 wall units $(1.5 \delta)$. The equivalent plot at $z / \delta=0.5\left(z^{+}=530\right)$ is much shorter, extending a distance of only $0.6 \delta$. These locations are chosen to show representative views of both the log region and wake region of the turbulent boundary layer. Additional correlation plots from intermediate planes suggest that the extent of the streamwise coherence drops off significantly beyond the log layer. This reduced streamwise coherence is clearly evident from the vector fields beginning at $z / \delta=0.2$ $\left(z^{+}=198\right)$, which typically contain evidence of larger individual vortex cores, upwash 
and downwash regions, and possibly cross-sections of individual hairpin heads (see Longmire, Ganapathisubramani \& Marusic 2001). Furthermore, the feature-detection algorithm mentioned earlier (Ganapathisubramani et al. 2003) did not identify any long packets when applied at $z / \delta=0.5$. The $R_{u u}$ measurements at $z / \delta=0.5$ appear very similar to the space-time correlation results of Kovasznay et al. (1970) obtained at a similar height $\left(z / \delta=0.45, z^{+}=580\right)$ in a boundary layer with similar $\operatorname{Re}_{\theta}$ and $R e_{\tau}$ (this was the closest wall-normal location in the previous study).

At $z^{+}=92$, the spanwise width of the positive correlation region is about 400 wall units, with a change in sign occurring at $\Delta y^{+}=200$. At $z / \delta=0.5$, however, the positive correlation is wider with the sign change shifted to $\Delta y^{+}=400$. This location is again consistent with the results of Kovasznay et al. (1970). The spanwise results demonstrate that typical flow structures increase in width with increasing wall-normal distance. At $z^{+}=92$, the long negative correlation outboard of the positive correlation region supports the notion of adjacent low- and high-speed zones extending in the streamwise direction. This result is entirely consistent with a hairpin vortex model, where the legs of an individual hairpin vortex become increasingly streamwise and elongated near the wall.

The long streamwise coherence at $z^{+}=92$ could be caused either by long zones of negative $u$, as observed in the packet structures, or by long zones of positive $u$ which are sometimes observed on either side of the packets. In order to investigate the relative contributions, lines of vectors in the streamwise direction were examined for the streamwise length between zero crossings. A zero crossing is defined as the location at which $u$ changes sign. The probability density is computed for positive and negative $u$ (streamwise velocity fluctuations) versus streamwise length (length is the distance over which the quantity remains above zero or below zero). This is similar to the zero-crossing method defined by Kailasnath \& Sreenivasan (1993).

Separate probability density functions are generated for lengths of positive and negative $u$ between zero crossings. The normalized difference between these functions is then defined as,

$$
\Xi(u)=\frac{\text { p.d.f. }(u>0)-\text { p.d.f. }(u<0)}{\text { p.d.f. }(u>0)+\text { p.d.f. }(u<0)} \times 100,
$$

and the result at $z^{+}=92$ is plotted in figure 4 . A negative value in the ordinate of the plot indicates that strips of negative $u$ occur more frequently than strips of positive $u$. Note that the streamwise length plotted $\left(\Delta x^{+}\right)$is limited by the field of view. The plot shows that shorter negative and positive $u$ excursions $\left(\Delta x^{+}<1400\right)$ occur with approximately equal probability. However, for excursions longer than 1500 wall units, those with negative $u$ occur significantly more frequently than those with positive $u$. This result suggests that the long positive streamwise correlations in $R_{u u}$ (see figure $3 a$ ) are dominated by long low-speed regions such as those noted to occur within vortex packets. To clarify this point, a second-order polynomial was fitted to the raw data. This second-order curve (shown as a solid line in figure 4) clearly reveals the trend discussed above.

The primary advantage of stereo PIV data is the availability of all three velocity components. Hence, it is possible to compute all possible velocity auto- and cross-correlations over the streamwise-spanwise plane. Figure 5 shows the $R_{v v}$ and $R_{w w}$ correlations at the two representative wall-normal locations, respectively. Both correlations are compact in both streamwise and spanwise directions, indicating that the spanwise and wall-normal velocity fluctuations are localized and do not possess an extended streamwise or spanwise coherence across the boundary layer. There is 


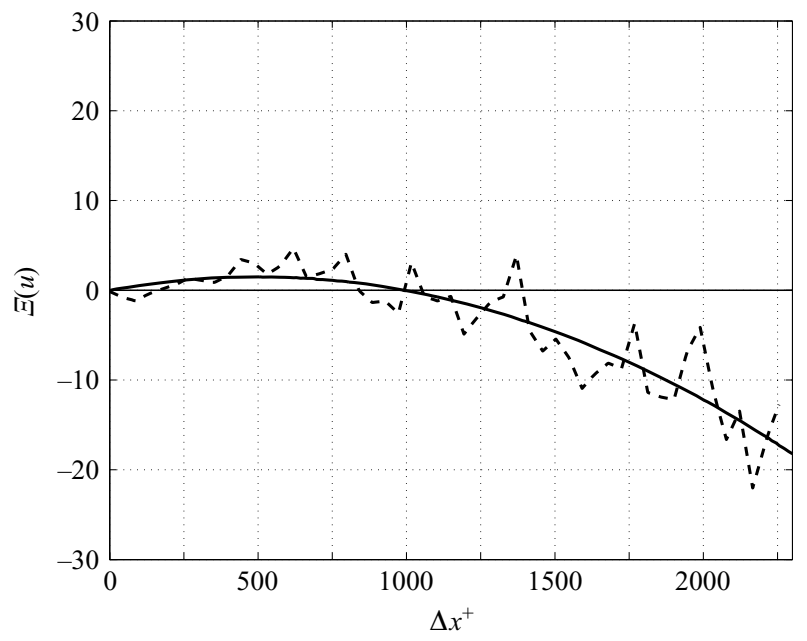

FigurE 4. The dashed line shows the difference between p.d.f. of positive and negative $u$ of a certain streamwise length. $\Delta x^{+}$is the streamwise distance over which the quantity remained above or below zero. The bin width chosen was 40 wall units. The solid line shows a second-order fit to the dashed line.

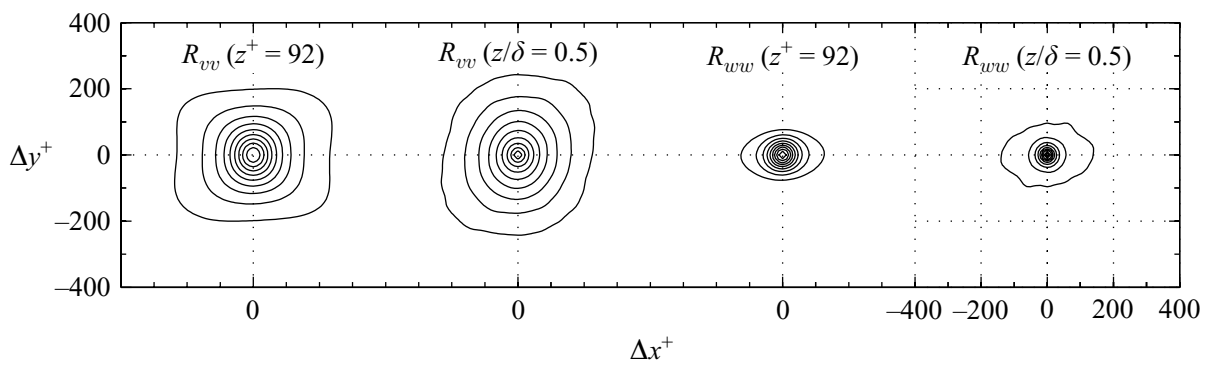

FIgURE 5. $R_{v v}$ and $R_{w w}$ correlations at $z^{+}=92$ and $z / \delta=0.5$ The contour levels range from 0.1 to 1 with increments of 0.1 .

small increase in the spanwise extent of both $R_{v v}$ and $R_{w w}$ correlations away from the wall, as seen in figure 5. This increase is attributed to the increase in geometric size of the representative average vortex structure. Also, note that the spanwise extent of $R_{v v}$ is larger than $R_{w w}$ in both locations. These results are in qualitative agreement with the findings of Krogstad \& Antonia (1994) who performed experiments using cross-wire arrays.

Figures $6(a)$ and $6(b)$ show the $R_{u w}$ correlation (streamwise-wall-normal) at $z^{+}=92$ and $z / \delta=0.5$, respectively. As with $R_{u u}$, the $R_{u w}$ correlation extends over a long streamwise distance at $z^{+}=92$, but the correlation values are negative for $\Delta y^{+}=0$. The maximum magnitude of $R_{u w}$ is about -0.37 . Given the result that low-speed events are primarily responsible for long correlations, the negative values of $R_{u w}$ in figure 6 suggest that slow-moving fluid is associated with upwash (flow away from the wall) over long streamwise distances. Further away from the wall at $z / \delta=0.5$, the $R_{u w}$ correlation decreases in length and increases in width similar to the behaviour of $R_{u u}$. Again, at this location, the size and shape of the spatial correlation is similar to that found by Kovasznay et al. (1970) from space-time correlations. The maximum magnitude of $R_{u w}$ at this position is -0.32 which is close to the correlation values 

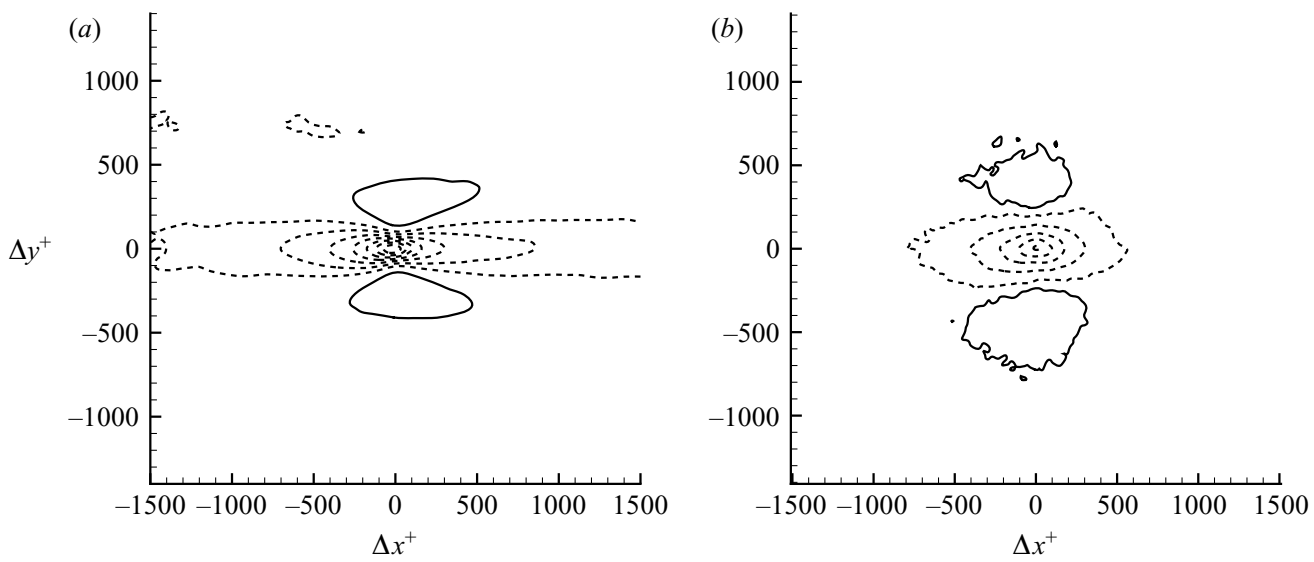

FIgURE 6. (a) $R_{u w}$ correlation at $z^{+}=92$, (b) $R_{u w}$ correlation at $z / \delta=0.5$. The contour levels for $R_{u w}$ range from 0.05 to -0.5 with spacing of 0.05 . Zero contours are not shown.

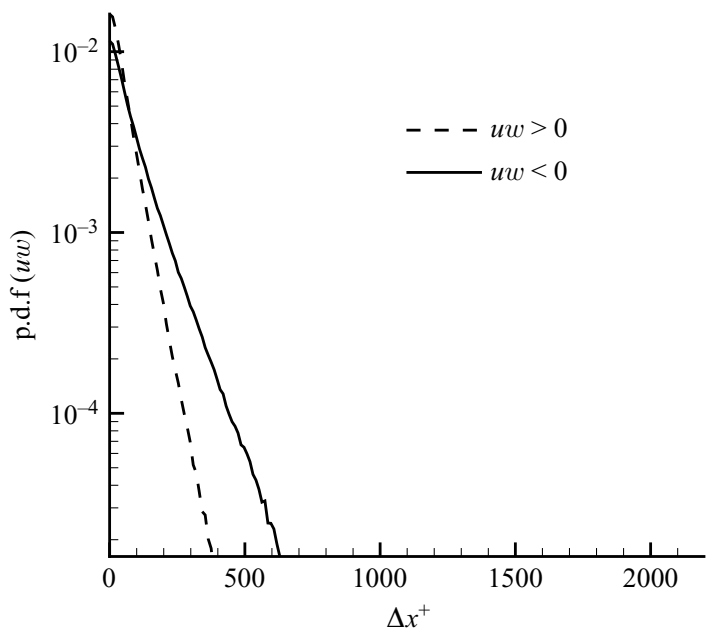

FIGURE 7. The probability density function of positive and negative $u w$ events. The p.d.f. ordinate is in log scale. $\Delta x^{+}$is the streamwise length over which the quantity remains above or below zero.

found in previous hot-wire experiments by Tritton (1967). One other point worth observing is the sign change of the $R_{u w}$ correlation in the spanwise direction. The presence of the sign change suggests that slow-moving fluid is correlated with flow towards the wall (downwash) at a distance of about 200 wall units on either side. This observation is in accordance with the inclined hairpin vortex theory (Theodorsen 1952; Perry \& Chong 1982; Perry \& Marusic 1995).

A zero-crossing analysis was also performed on the $u w$ product at $z^{+}=92$. Similar to the case of $u$, separate probability density functions were determined for the length of streamwise strips between zero crossings for both $u w<0$ and $u w>0$. The resulting p.d.f.s are plotted in figure 7. These distributions are narrower than those based on $u$ generated for figure 4, which can be expected based on the correlation plots. The distribution of $u w<0$ is broader than that of $u w>0$, indicating that $u w<0$ is associated with slightly longer structures. This result might be explained 

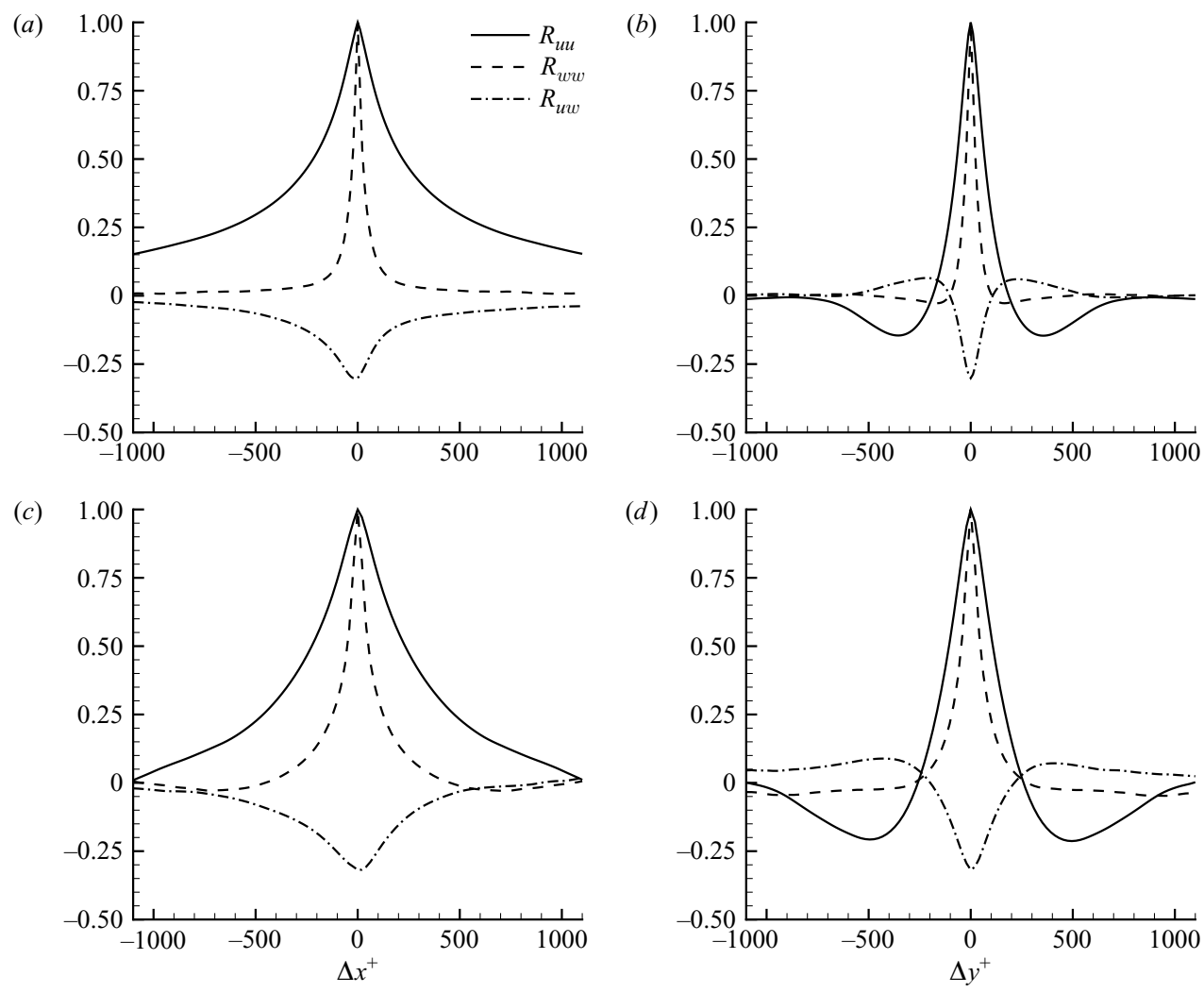

FiguRE 8. $R_{u u}, R_{u w}$ and $R_{w w}$ correlations. For $z^{+}=92,(a)$ streamwise direction at $\Delta y^{+}=0$ and $(b)$ spanwise direction at $\Delta x^{+}=0$. For $z / \delta=0.5,(c)$ streamwise direction at $\Delta y^{+}=0$ and (d) spanwise direction at $\Delta x^{+}=0$.

by the argument that $u w<0$ events derive naturally from angled eddies generating upwash and downwash while $u w>0$ events are more random. This is consistent with all existing literature on Reynolds shear stress quadrant analysis (see Lu \& Willmarth 1973). It was found that the Q2 and Q4 events always dominate the Q1 and Q3 events throughout the boundary layer which explains $\overline{u w}<0$. When $u w<0$ was broken down into separate zero-crossing length distributions for Q2 $(u<0)$ and Q4 $(u>0)$, no significant difference was observable (and therefore the separate distributions are not shown in the plot). This is also in agreement with the results of Willmarth \& Lu (1972) who show that Q2 and Q4 contributions are of similar strength.

In figure $8(a)$, the $R_{w w}$ correlation at $z^{+}=92$ is compared with $R_{u u}$ and $R_{u w}$ results along the centreline in the streamwise direction. First, note that the $R_{w w}$ correlation drops very sharply when $\Delta x^{+} \leqslant 100$, suggesting that the streamwise coherence in $w$ is typically quite short as would occur for ejections associated with individual hairpins. On the other hand, the peak in $R_{u w}$ drops off more slowly, and the correlation has significant magnitude over the entire range plotted and beyond. (The normalized correlation magnitude for $\Delta x^{+}=2000$ is -0.034 , or $11 \%$ of the peak value). The $R_{u u}$ correlation extends beyond the streamwise range examined, clearly indicating the presence of streamwise regions longer than $2 \delta$. Taken together, these results suggest that slow-moving fluid is correlated with discrete zones of upwash over an extended 
streamwise distance both upstream and downstream, again as would be expected for packets of hairpin vortices moving with similar convection speeds. Note that the $R_{u w}$ correlation is slightly asymmetric in the streamwise direction, such that the correlation has a longer tail for positive $\Delta x^{+}$than for negative $\Delta x^{+}$. This can be explained by the following argument. Typically, vortex packet structures have fairly uniform streamwise velocity over their streamwise extent (see Ganapathisubramani et al. 2003). On the other hand, the results of Adrian et al. (2000) suggested that the hairpins at the downstream end of the packet tend to be larger than those at the upstream end, leading to stronger positive values of $w$ at the downstream end. The resulting $R_{u w}$ values for large positive $\Delta x^{+}$should thus be larger than for large negative $\Delta x^{+}$.

The spanwise extent of the structures is shown in figure 8(b) in which $R_{u u}, R_{u w}$ and $R_{w w}$ are compared in the $y$-direction at $\Delta x=0$. The $R_{u u}$ correlation in the spanwise direction drops from 1 to a negative value at $\Delta y^{+} \approx 200$. The spanwise $R_{u u}$ also indicates a general trend where the value drops below zero on either side of the peak, indicating the occurrence of alternating high-speed and low-speed regions in the spanwise direction. The $R_{u w}$ correlation changes sign from negative to positive at around the same spanwise displacement as $R_{u u}$, suggesting that the Reynolds shear stress production has a very similar spanwise scale to the streamwise velocity. Figure $8(b)$ also shows that the $R_{w w}$ correlation is narrower in the spanwise direction than $R_{u u}$, and that its negative lobe is weaker, suggesting, in general, that coherence in $w$ is narrower than in $u$. In any case, the presence of negative lobes in $R_{w w}$ suggests that upwash locations are frequently bounded by downwash, as would occur in hairpin vortices where an ejection between angled legs would be accompanied by inward motion beneath the legs. The streamwise and spanwise extents of the correlations further from the wall $(z / \delta=0.5)$ are depicted in figures $8(c)$ and $8(d)$. It is evident from these two figures that the $R_{u u}$ correlations are shorter in the streamwise direction and broader in the spanwise direction than at $z^{+}=92$, indicating a reduced streamwise and increased spanwise spatial coherence.

Tomkins \& Adrian (2003) found that an eddy conditioned on slow-moving zones in the streamwise-spanwise plane bore a striking resemblance to a hairpin vortex signature. Similar to the present results, they also found that the spanwise width of the structure and the separation between the legs increased away from the wall which is analogous to the increase in the spanwise extent of the $R_{u u}$ correlations.

It is worth mentioning that the present results do not agree with some of the trends found by Christensen (2001) who performed spatial correlations of streamwise-wallnormal PIV data taken in a turbulent channel flow with very similar $R e_{\tau}$ to the present study $\left(R e_{\tau}=1141\right)$. Christensen reported $R_{u u}, R_{w w}$ and $R_{u w}$ values with no spanwise displacement at various wall-normal positions including $z^{+}=103(z / h=0.09$, where $h$ is the channel half-width). Within the Christensen data, $R_{u u}$ and $R_{u w}$ were elongated in the streamwise direction compared with $R_{w w}$, as in the present data. Christensen's fields covered $\Delta x$ values up to $0.72 h$, representing a shorter streamwise range than the present data. Nevertheless, direct comparison of like contour levels shows that the channel data exhibit longer correlations at $z^{+}=103(z / h=0.09)$ than the present boundary-layer data at $z^{+}=92(z / \delta=0.09)$. In addition, the length of the channel correlations increased with wall normal distance in contrast to the length of the present boundary-layer correlations which decreased from the log to the wake region. Perhaps, differences in boundary layer and channel results are not surprising, given that fully developed channel flow includes additional structures initiated on the opposite wall that can influence the measurement regions. This additional influence 
from the opposite wall in low-Reynolds-number flows was documented by various workers. Wei \& Willmarth (1989) suggested that opposite near-wall regions constantly exchange vorticity and Reynolds shear stress while Antonia et al. (1992) investigated this phenomenon (in experiment and simulation) by introducing temperature increases on one wall and detecting it on the opposite wall. They found that fluid from the slightly heated wall region can reach the near-wall region of the opposite wall. The penetration depth and frequency of occurrence of thermal excursions decreases with Reynolds number. In a channel flow at a fairly low Reynolds number, therefore, no outer region exists within which the turbulence generated by the wall-shearing dies out (as occurs in an isolated boundary layer).

If a contour level of $R_{u u}$ (say 0.5 ) is chosen to define a representative streamwise length scale $\left(l_{x}\right)$, the present results reveal an increase in $l_{x}$ through the log region (between $z^{+}=92$ and 150), but then a decrease in $l_{x}$ in the outer wake region (at $z / \delta=0.2$ and 0.5 ). Further work to complement present results would clarify this increasing trend through the log region. However, Christensen's channel flow data clearly show increasing streamwise length scales throughout the log region and it should be noted that the entire outer region in a channel flow is effectively a log region (as evidenced in the mean velocity profiles in Christensen 2001, p. 61) and there is no intermittency as would be found in the outer region of a boundary layer.

\subsection{Comparisons with inclined cross-stream PIV data}

Figure 9 shows the streamwise-spanwise $R_{u u}$ correlations along with those produced from the inclined cross-stream planes for wall-normal location $z^{+}=92$. For clarity, the $45^{\circ}$ and $135^{\circ}$ planes are shown separately in figures $9(a)$ and $9(b)$. Figure $9(c)$ is a plan view of all three planes together. It is clear from these figures that the long streamwise correlations in the streamwise-spanwise planes also extend in the wall-normal direction. The wall-normal extent of the correlations is longer in the $45^{\circ}$ plane than the $135^{\circ}$ plane. Later in this section, this asymmetry will be quantified and discussed.

In order to judge the quality of the match between the correlations from the two datasets, we can extract the line of data along the intersection of the planes at $\Delta x^{+}=0$. Figure 10(a) shows the extracted profile of $R_{u u}$ from the streamwise-spanwise and $45^{\circ}$ planes at $z^{+}=92$. It is clear from this plot that the correlations from the two datasets match very well close to the origin, with only a slight deviation at larger $\Delta y^{+}$. This mismatch away from the origin is attributed to poorer convergence of the correlation function calculations in the inclined plane datasets. Previous literature (Christensen 2001) emphasizes the need for more than 3000 independent realizations of PIV data for convergence within $1 \%$. A threshold $t h=0.05$, as shown in figure $10(a)$ is defined to quantify a spanwise length scale $\left(l_{y}\right)$ based on $R_{u u}$. This spanwise length scale is computed across the complete range of wall-normal locations and the results are plotted in figure $10(b)$. The curves show the data from the inclined planes, and the solid symbols show the data points from the $(x, y)$-plane. Aside from the obvious good agreement between datasets, this plot also indicates a linear increase in the spanwise length scale through the log region (up to $z^{+} \sim 200$ ) and what appears to be a smaller linear increase through the wake region. The linear trend in the log region was previously observed by Tomkins \& Adrian (2003) in their streamwise-spanwise PIV data.

Figure 11( $a$ ) shows a line plot of $R_{u u}$ computed with $z^{+}{ }_{r e f}=92$ along an inclined wall-normal line $z^{\prime}$, at $\Delta y^{+}=0$ (marked in figure $9 c$ ). The correlations are computed in the inclined plane $\left(y^{\prime}-z^{\prime}\right)$ with a $z^{\prime}$ reference location equivalent to $z^{+}=92$. The 

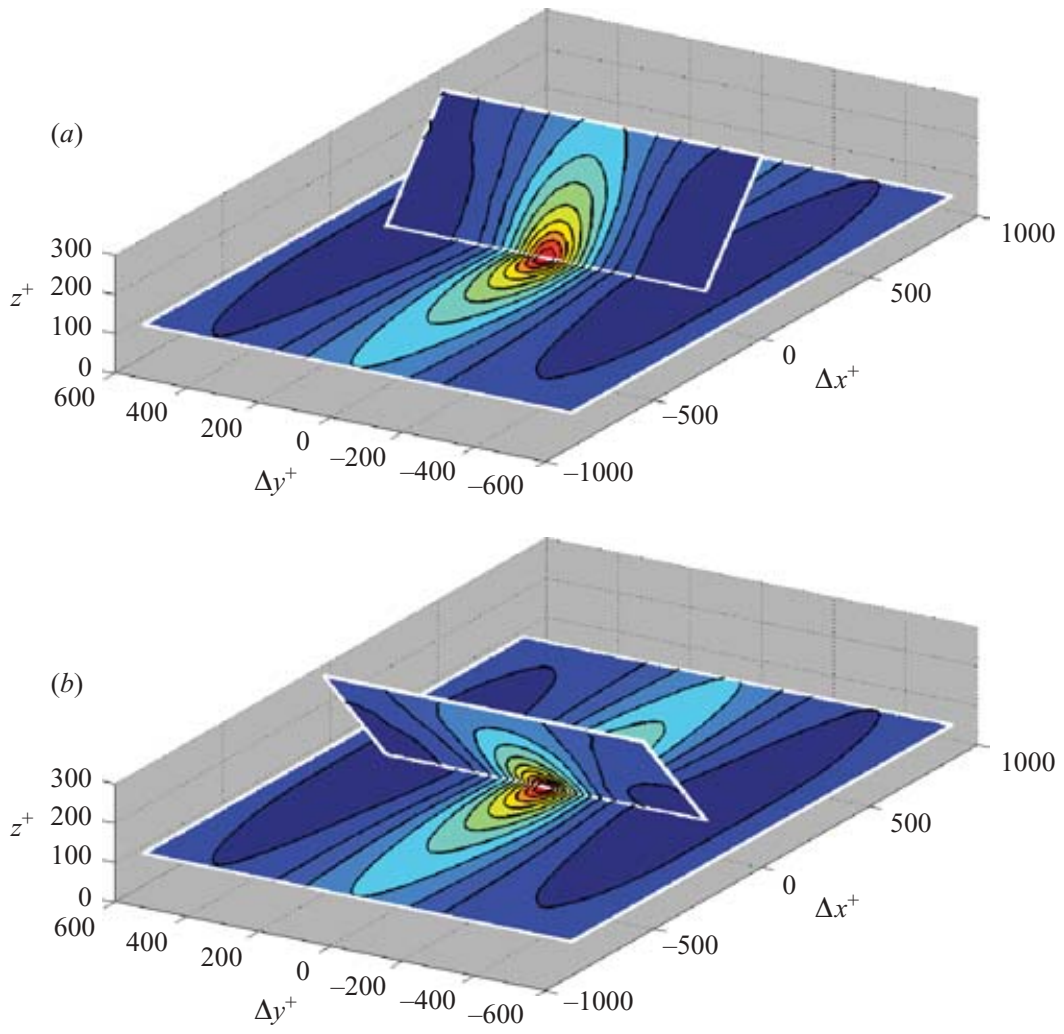

(c)
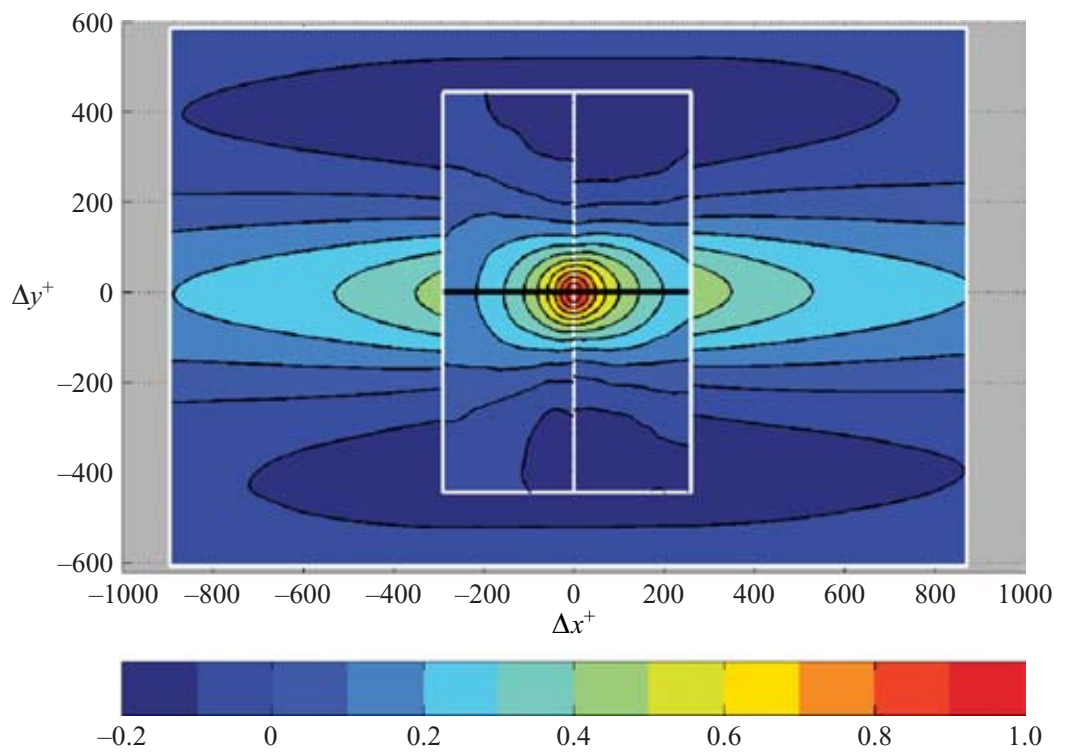

FIGURE 9. $R_{u u}$ correlation at $z_{r e f}^{+}=92$. Perspective view of $(x, y)(a)$ and $45^{\circ}$ planes, $(b)$ and $135^{\circ}$ planes stacked together. (c) Top view of all three planes together. The white lines show the edge of the correlation box size. The flow is along the positive $x$-direction. 

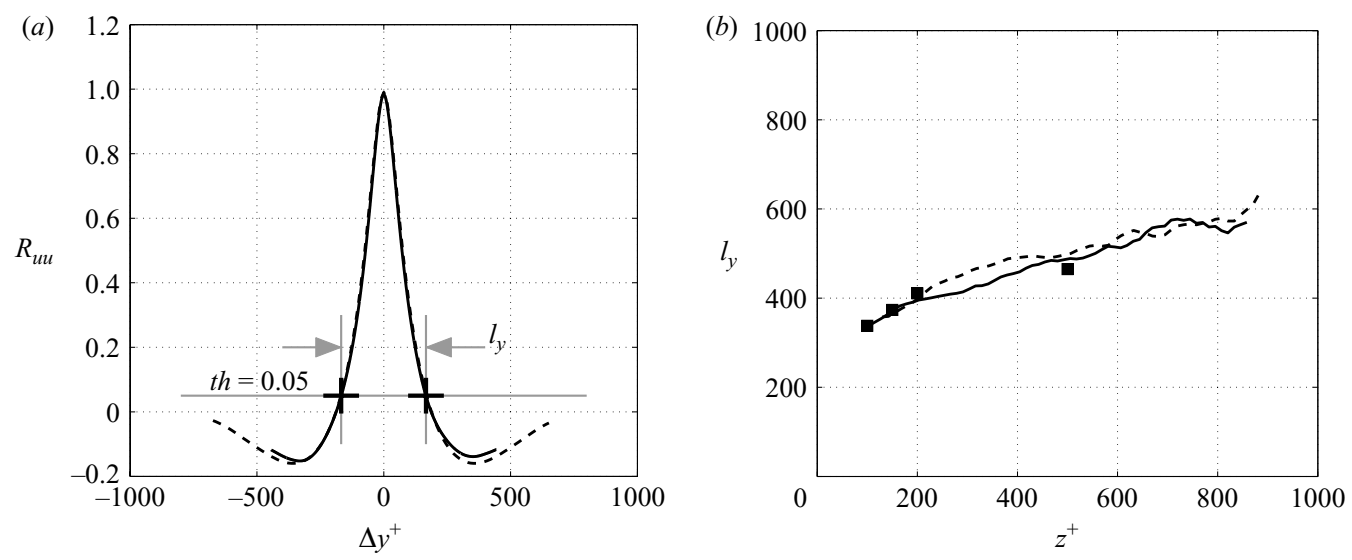

Figure 10. (a) Spanwise line plot of $R_{u u}$ at $z^{+}=92$ from the two datasets, the solid line shows the $(x, y)$-plane and dashed line shows the $45^{\circ}$ plane. $(b)$ Spanwise length scale $\left(l_{y}\right)$ as a function of the wall-normal location. The solid line represents the $45^{\circ}$ plane, the dashed line the $135^{\circ}$ plane and the symbols are for the streamwise-spanwise plane.
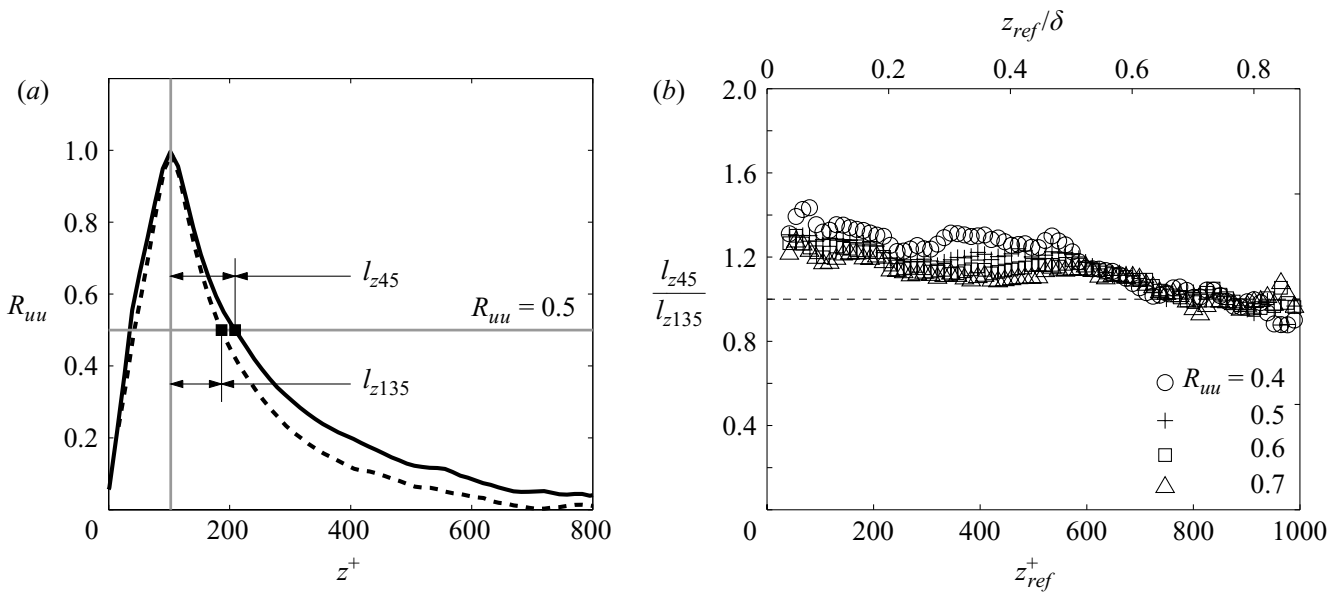

FiguRE 11. (a) Line plot of $R_{u u}$ at $z_{\text {ref }}^{+}=92$ along the wall-normal direction at $\Delta y^{+}=0$. (b) The ratio of $l_{z 45}$ to $l_{z 135}$ for all $z_{r e f}$ across the boundary layer. Symbols show the ratio $l_{z 45} / l_{z 135}$, with length scales computed using various contour levels.

abscissa in the figure shows actual wall-normal distance $z$, computed from $z^{\prime}$ using simple trigonometry. The figure reveals the asymmetry of $R_{u u}$ in the wall-normal extent for the $45^{\circ}$ and $135^{\circ}$ planes, clearly indicating that the correlation is longer in the $45^{\circ}$ plane. For example, consider the 0.5 contour levels for the two cases and define two length scales $l_{z 45}$ and $l_{z 135}$ for the the $45^{\circ}$ and $135^{\circ}$ planes, respectively, based on the chosen contour level as marked by the horizontal line in figure 11 $(a)$. It is clear that $l_{z 45}$ would extend to a higher wall-normal position than $l_{z 135}$. This observation is also clear in figure $11(b)$ where the ratio of $l_{z 45}$ to $l_{z 135}$ for various contour levels is plotted for the full wall-normal range of the inclined plane data. The ratio is consistently greater than 1 for all the contours used to compute the length scales. This feature is in agreement with Christensen (2001), who performed PIV experiments in streamwise-wall-normal planes in a channel flow. Christensen's 


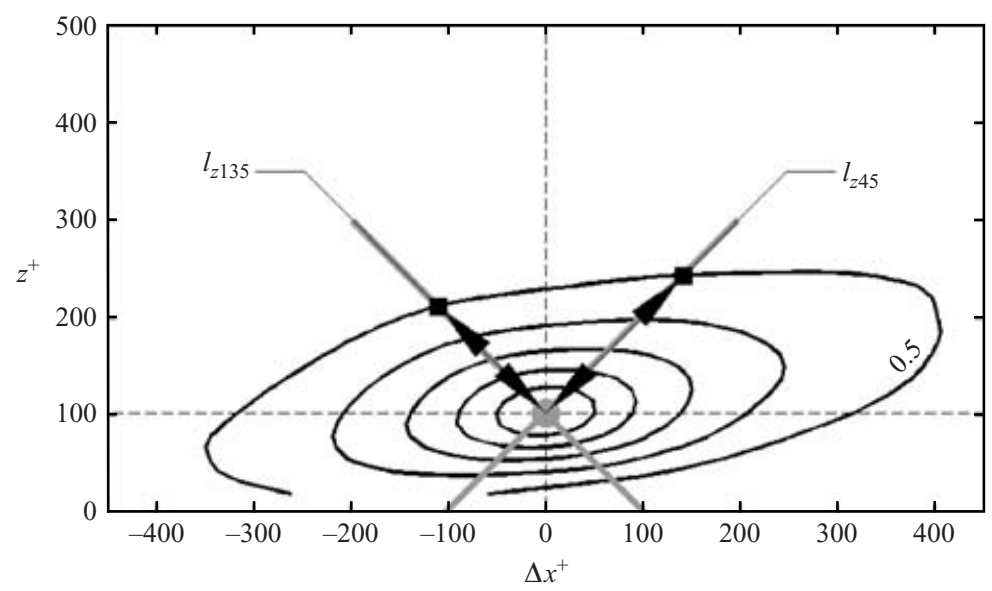

FigURE 12. $R_{u u}$ from Christensen (2001) at $z_{\text {ref }}^{+}=102.6$.

data is reproduced in figure 12 which shows contours of $R_{u u}$ at a reference height of $z^{+}{ }_{r e f}=103$ for $R e_{\tau}=1141$. Representations of the inclined $45^{\circ}$ and $135^{\circ}$ planes are included in the figure. It is clear from the intersection of these lines with the 0.5 contour lines that $l_{z 45}$ would indeed be expected to be longer than $l_{z 135}$. Although the percentage difference between $l_{z 45}$ and $l_{z 135}$ is similar for both the present inclined plane data and the Christensen (2001) results $\left(l_{z 45}\right.$ is $25 \%$ larger than $\left.l_{z 135}\right)$, there is some disparity in the actual values. Both length scales are longer in the channelflow data. This difference is attributed to the aforementioned fundamental differences between turbulent-channel and boundary-layer flows.

Figure $11(b)$ also indicates that the $l_{z 45}$ is about $25-40 \%$ greater than $l_{z 135}$ through the $\log$ region. In this region, the ratio is higher for lower contour levels. The plot reveals a decreasing trend in the ratio across the boundary layer. The ratio tends to 1 towards the edge of the outer wake region for all contour levels. This result is not surprising considering the intermittency in the outer wake region. Nearly all structures reaching the edge of the boundary layer are likely not to be inclined and, hence, have equal contributions in $45^{\circ}$ and $135^{\circ}$ planes.

The fact that $l_{z 45}$ is greater than $l_{z 135}$ is clearly indicative of the inclined structure behaviour seen by Favre et al. (1957) and others in their correlation measurements using hot wires and exhibited in Christensen (2001) and Christensen \& Adrian (2001). The asymmetry in the length scales can be explained using the hairpin packet paradigm. Figure 13 is a schematic of a hairpin packet and associated low-speed region. Considering two planes at $45^{\circ}$ and $135^{\circ}$ cutting across this packet as shown, it is clear that the $45^{\circ}$ planes will encounter a longer region of low-speed flow in the $z^{\prime}$-direction than the $135^{\circ}$ plane. Clearly, there is an inclined ramp-like shape associated with the idealized hairpin packet that would manifest itself in the $R_{u u}$ contours.

Alternatively, if we consider that there exists a nested hierarchy of hairpin packets as proposed by Adrian et al. (2000), shown in figure 22 of their paper, then such an arrangement assumes that packets of hairpin vortices in the outer wake region skip over those in the log region. However, in this scenario, the individual hairpins in the outer wake region are spaced farther apart in the streamwise direction (as shown clearly in their figure) and, hence, will not necessarily be identified as a packet by the algorithm described in Ganapathisubramani et al. (2003). The present result, $l_{z 45}$ 


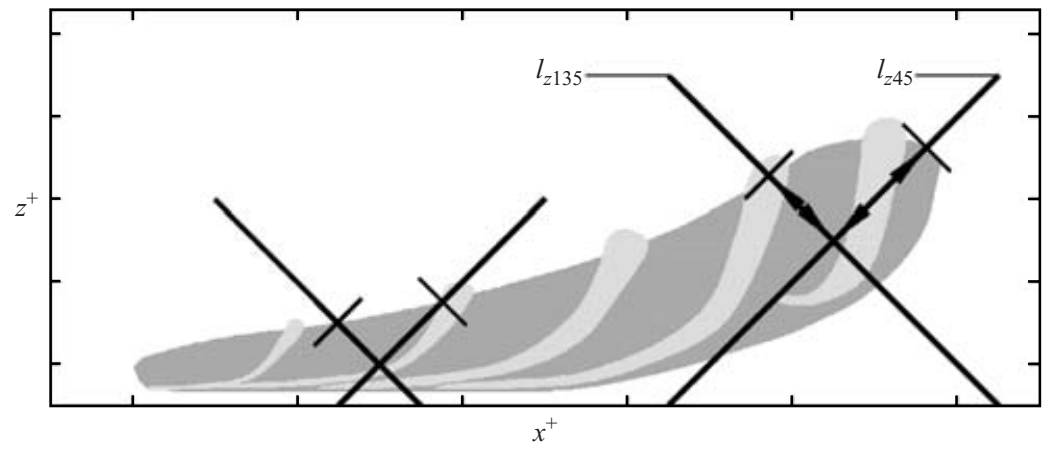

FIGURE 13. Schematic of a lifted hairpin packet and its associated low-speed region.

greater than $l_{z 135}$, can be explained alternatively using this nested hierarchy of hairpin packets as well. If $45^{\circ}$ and $135^{\circ}$ lines are drawn in their figure showing the nested arrangement, it would clearly show that the $45^{\circ}$ planes will detect a longer region of low-speed flow in the $z^{\prime}$-direction than the $135^{\circ}$ plane.

Having established the quality of match in the correlations between the streamwisespanwise and the inclined planes in figure 10, a few select contour levels can be extracted to further elaborate the structure of the boundary layer. Figure 14 shows skeleton plots of two $R_{u u}$ contour levels (0.3 and 0.4$)$ in all three available planes at two wall-normal locations. The streamwise extent of the contours are long in the $\log$ region $\left(z^{+}=92\right.$ ). It was noted (not shown) that the streamwise length of the 0.3 contour level increases within the log region, between $z^{+}=92$ and $z^{+}=150$ and decreases away from the wall in the wake region.

The most significant observation from these plots is the shape of the contour in the inclined planes. The contours are more distorted and flattened near the wall in both $45^{\circ}$ and $135^{\circ}$ planes in the log region (figure $14 a$ ) whilst exhibiting a more symmetric shape in the outer wake region (figure 14b). In order to clarify this point, figures $15(a)$ and $15(b)$ show a projection of the 0.3 contour level onto the spanwise-wall-normal plane at all four reference heights, for both the $45^{\circ}$ and $135^{\circ}$ planes, respectively. It is noted that the contours in the log region are definitely flatter near the wall in both inclined planes. The contour at $z / \delta=0.2$ and 0.5 (which are in the outer wake region) have a smoother and more elliptical profile, that appears symmetric about $z_{\text {ref }}$. The flattened contour shapes for $z_{\text {ref }}$ within the log region indicate that the velocity fluctuations at these wall-normal locations are 'coupled' with those occurring near the wall (buffer region). Conversely, the fact that the comparable contour levels in the outer wake region are rounded and relatively far from the wall implies that velocity fluctuations beyond the log region are 'separated' from those occurring near the wall (statistically uncorrelated).

The above observation can also be rationalized using the hairpin-packet model. Referring to the two sets of inclined planes drawn in figure 13, the pair on the left of the figure cross at a reference point in the log region. In this case, the planes encounter low-speed regions that extend all the way to the wall which, of course, will be reflected in the $R_{u u}$ contours. However, if the reference point is moved higher into the wake region (shown by the planes on the right-hand side of the figure), only the uppermost portion of the packet can contribute to the correlation. At such large $z_{\text {ref }}$, it is clear that the low-speed region associated with the packet does not extend to the wall and, hence, neither will the $R_{u u}$ contours. Similar arguments apply also if we 

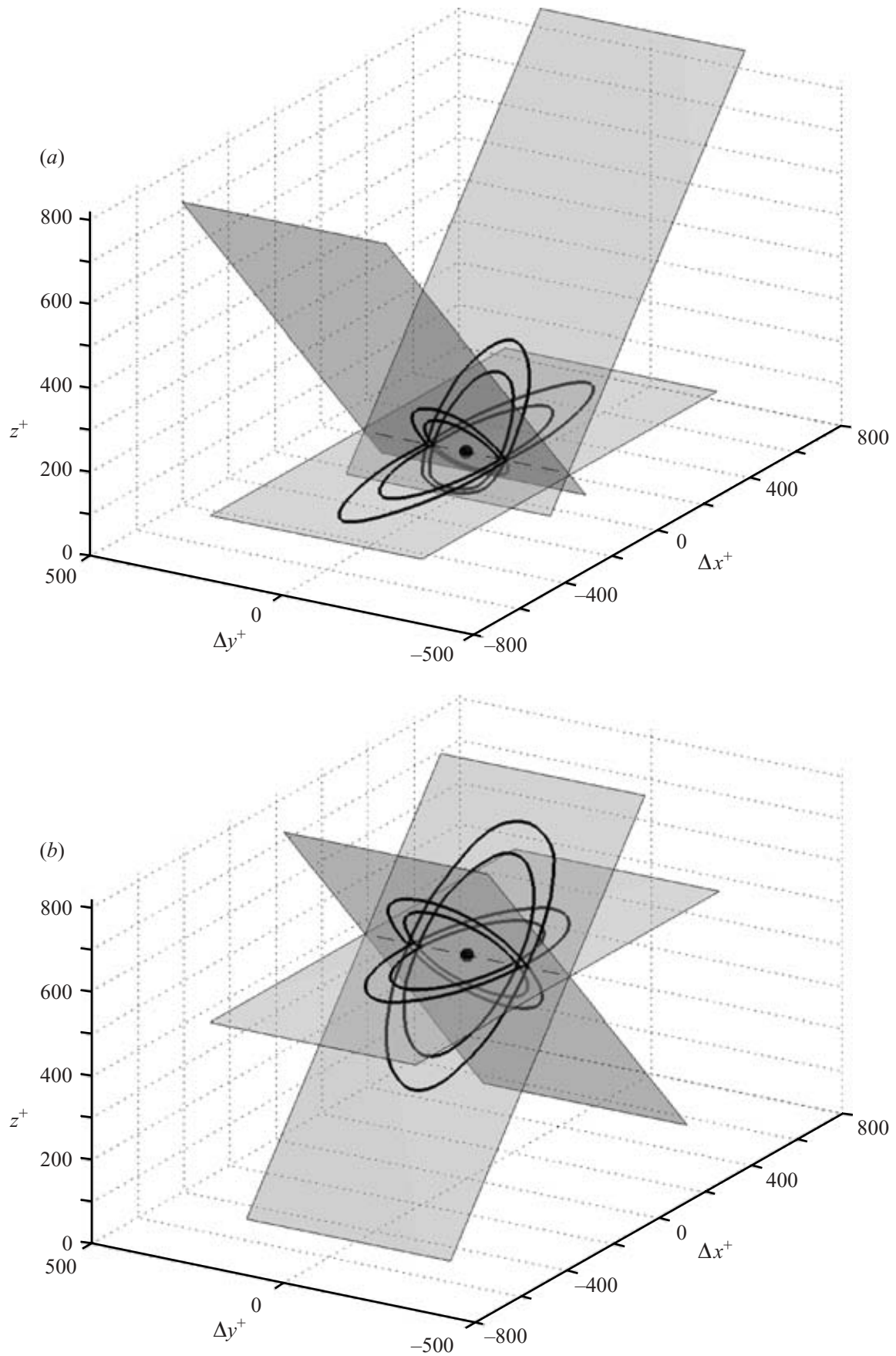

Figure 14. Planar $R_{u u}$ contours at 0.3 and 0.4 , shown for three available datasets $((x, y)$, $45^{\circ}$ and $135^{\circ}$ planes). (a) $z_{\text {ref }}^{+}=92,(b) z_{\text {ref }}^{+}=530(z / \delta=0.5)$.

consider the scenario where a nested arrangement of hairpin packets described by Adrian et al. (2000) exists, where packets in the outer wake region are skipping over packets in the log region. If a pair of inclined planes crossing at a reference point in the log region in the nested packet arrangement is considered, the low-speed region 

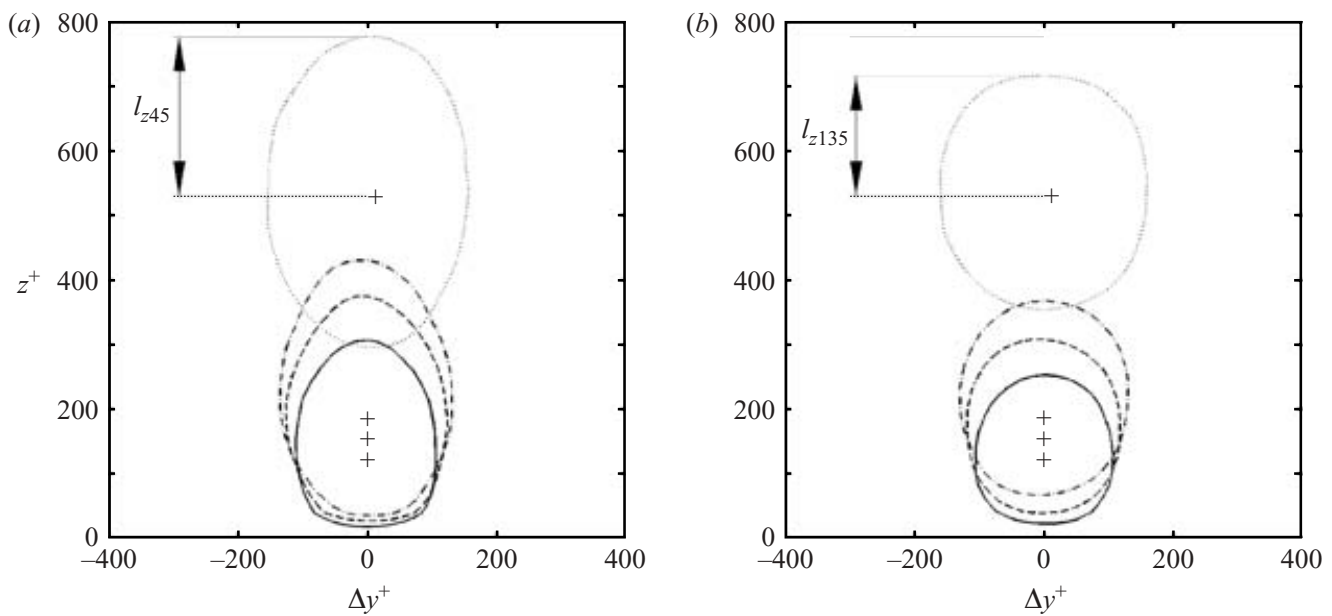

FIGURE 15. Projections of the skeleton plot for $R_{u u}=0.3$ on to the spanwise-wall-normal plane. (a) $45^{\circ}$ plane data and $(b) 135^{\circ}$ plane with all reference points. The solid line shows $z_{\text {ref }}^{+}=92$, dashed lines show $z_{\text {ref }}^{+}=150$, dashed-dot lines show $z_{\text {ref }}=0.2 \delta$ and dotted lines show $z_{\text {ref }}=0.5 \delta$. The ordinate in the figures is actual wall-normal distance calculated using trigonometry.

associated with the packet extends all the way to the wall. However, if the reference point is moved to the outer wake region, the low-speed region associated with the packet does not reach the wall and this will manifest itself into $R_{u u}$ contours which will not have comparable correlation near to the wall.

Figure 13 also clarifies the point that $l_{z 45}$ is longer than $l_{z 135}$ at either end of the idealized packet. A comparison between equivalent contours in figures $15(a)$ and $15(b)$ reinforces this view that $l_{z 45}$ is greater than $l_{z 135}$ for all four $z_{\text {ref }}$ contours shown. Hence, the inclined tendency of the correlated structure persists through the log region and into the wake region. However, figure 11(b) indicated that this inclined tendency gradually diminishes towards the outer edge of the boundary layer.

\section{Conclusions}

In this paper, two-point correlation functions computed from stereo PIV data obtained in streamwise-spanwise planes and inclined cross-stream planes (inclined at $45^{\circ}$ and $135^{\circ}$ to the streamwise direction) are presented and interpreted. The streamwise velocity fluctuation correlations $\left(R_{u u}\right)$ computed from the two different datasets are in good agreement. The $R_{u u}$ correlation from the $(x, y)$ and inclined $45^{\circ}$ and $135^{\circ}$ planes computed for the corresponding wall-normal reference heights $z_{\text {ref }}^{+}$ are stacked together to gauge the streamwise, spanwise and wall-normal coherence of the velocity fluctuations. The important conclusions resulting from the investigation of these correlations are as follows.

(a) The streamwise-spanwise $R_{u u}$ and $R_{u w}$ correlations suggest that, in the log region, long narrow zones with uniform speed contain intermittent zones of upwash or downwash over their length, as found previously by other workers (Tritton 1967; Kovasznay et al. 1970; Bogard \& Tiederman 1986; Tardu 1995) using pointmeasurement techniques and Taylor's hypothesis. The data also seem to indicate an increase in the streamwise length scale through the log region (between $z^{+}=92$ and 150 ) and then a decreasing trend in the outer wake region (between $z / \delta=0.2$ and 0.5 ). 
These conclusions are based on only a few points, and therefore it would be useful to test the trend in the log region with future work. The spanwise length scale $\left(l_{y}\right)$ clearly grows with increasing wall-normal distance. In general, the rate of growth is found to be approximately linear across the boundary layer, with the possible indication that the growth rate is higher in the log region than the wake region.

(b) Investigation of the zero-crossing events indicates that long low-speed zones are statistically more significant than high-speed zones in the log region, and, hence, are the more significant contributors to the tail of $R_{u u}$ correlations. The fact that $R_{u w}$ is negative at $\Delta y^{+}=0$, suggests that these elongated low-speed zones are associated with regions of upwash. However, the compact nature of $R_{w w}$ correlations indicates that these regions of upwash are spatially compact. This picture is consistent with the idea that packets of hairpin vortices dominate the long streamwise coherence in the correlations in the log region.

(c) Simultaneous visualization of $R_{u u}$ in three different planes reveals the extent of the velocity coherence in all three directions. $R_{u u}$ is longer along the $45^{\circ}$ incline than the $135^{\circ}$ incline, analogous to a hairpin packet dragging a forward-inclined low-speed region as shown in figure 14(a). This result is in general agreement with the correlation measurements performed by Christensen (2001) in channel flow.

(d) A closer examination of the $R_{u u}$ correlations in the $45^{\circ}$ and $135^{\circ}$ planes with reference points closer to the wall, show contours flattening near the wall. For reference points in the wake region, the contours have a symmetric oval shape, suggesting that most velocity fluctuations in the wake region are independent of the fluctuations closer to the wall. This can be explained by the concept that hairpin packets are inclined and do not extend all the way to the wall towards the leading edge. This could also be indicative of a nested hierarchy of packets across the boundary layer, as proposed by Adrian et al. (2000). Either way, the correlated events in the wake region appear localized in the wall-normal direction, implying that the velocity signature associated with the larger hairpins or hairpin packets does not extend to the wall.

The authors gratefully acknowledge support from the National Science Foundation through Grants ACI-9982274, CTS-9983933 and CTS-0324898, the Graduate school of the University of Minnesota and the David and Lucile Packard Foundation. The authors also thank Professor Christensen for making available his channel-flow data.

\section{REFERENCES}

ACARlar, M. S. \& SMith, C. R. 1987a A study of hairpin vortices in a laminar boundary layer. Part 1. Hairpin vortices generated by a hemisphere protuberance. J. Fluid Mech. 175, 1-41.

ACARlaR, M. S. \& SMith, C. R. $1987 b$ A study of hairpin vortices in a laminar boundary layer. Part 2. Hairpin vortices generated by fluid injection. J. Fluid Mech. 175, 43-83.

Adrian, R. J., Meinhart, C. D. \& Tomkins, C. D. 2000 Vortex organization in the outer region of the turbulent boundary layer. J. Fluid Mech. 422, 1-53.

Antonia, R. A., Teitel, M., Kim, J. \& Browne, L. W. B. 1992 Low Reynolds number effects in a fully developed channel flow. J. Fluid Mech. 236, 579-605.

BANDYOPADHYAY, P. 1980 Large structure with a characteristic upstream interface in turbulent boundary layers. Phys. Fluids 23, 2326-2327.

BLACKWELDER, R. F. \& KAPlAN, R. E. 1976 On the wall structure of the turbulent boundary layer. J. Fluid Mech. 76, 89-112.

Bogard, D. G. \& Tiederman, W. G. 1986 Burst detection with single-point velocity measurements. J. Fluid Mech. 162, 389-413.

Brown, G. R. \& Thomas, A. S. W. 1977 Large structure in a turbulent boundary layer. Phys. Fluids 20, S243-S251. 
Christensen, K. T. 2001 Experimental investigation of acceleration and velocity fields in turbulent channel flow. PhD thesis, Department of Theoretical and Applied Mechanics, University of Illinois at Urbana-Champaign.

Christensen, K. T. \& Adrian, R. J. 2001 Statistical evidence of hairpin vortex packets in wall turbulence. J. Fluid Mech. 431, 433-443.

Corino, E. R. \& Brodkey, R. S. 1967 A visual investigation of the wall region in turbulent flow. J. Fluid Mech. 37, 1-30.

Favre, A. J., Gaviglio, J. J. \& Dumas, R. J. 1957 Space-time double correlations and spectra in a turbulent boundary layer. J. Fluid Mech. 2, 313-342.

Ganapathisubramani, B., Longmire, E. K. \& Marusic, I. 2002 Investigation of three dimensionality in the near field of a round jet using stereo PIV. J. Turbulence 3, 017.

Ganapathisubramani, B., Longmire, E. K. \& Marusic, I. 2003 Characteristics of vortex packets in turbulent boundary layers. J. Fluid Mech. 478, 35-46.

Grant, H. L. 1958 The large eddies of a turbulent motion. J. Fluid Mech. 4, 149-190.

Hama, F. R., Long, J. D. \& Hegarty, J. C. 1957 On transition from laminar to turbulent flow. J. Appl. Phys. 28, 388-394.

Head, M. R. \& Bandyopadhyay, P. 1981 New aspects of turbulent boundary-layer structure. J. Fluid Mech. 107, 297-337.

Hutchins, N., Hambleton, W. T. \& Marusic, I. 2004 Inclined cross-stream stereo PIV measurements in turbulent boundary layers. J. Fluid Mech. Submitted.

Kailasnath, P. \& Sreenivasan, K. R. 1993 Zero crossings of velocity fluctuations in turbulent boundary layers. Phys. Fluids 5, 2879-2885.

Kline, S. J., Reynolds, W. C., Schraub, F. A. \& Rundstadler, P. W. 1967 The structure of turbulent boundary layers. J. Fluid Mech. 30, 741-773.

Kovasznay, L. S. G., Kibens, V. \& Blackwelder, R. F. 1970 Large-scale motion in the intermittent region of a turbulent boundary layer. J. Fluid Mech. 41, 283-326.

Krogstad, P.-A. \& Antonia, R. A. 1994 Structure of turbulent boundary layers on smooth and rough walls. J. Fluid Mech. 277, 1-21.

Longmire, E. K., Ganapathisubramani, B. \& Marusic, I. 2001 Structure identification and analysis in turbulent boundary layers by stereo PIV. In Proc. 4th Intl Symp. on Particle Image Velocimetry, Sept. 17-19. Gottingen, Germany.

Lu, S. S. \& Willmarth, W. W. 1973 Measurements of the structure of the Reynolds stress in a turbulent boundary layer. J. Fluid Mech. 60, 481-511.

Marusic, I. 2001 On the role of large-scale structures in wall turbulence. Phys. Fluids 13, 735-743.

Marusic, I. \& Perry, A. E. 1995 A wall-wake model for the turbulence structure of boundary layers. Part 2. Further experimental support. J. Fluid Mech. 298, 389-407.

MoIn, P. \& KIm, J. 1985 The structure of the vorticity field in turbulent channel flow. Part 1. Analysis of instantaneous and statistical correlation. J. Fluid Mech. 155, 441-464.

Na, Y., Hanratty, T. J. \& LiU, Z. 2001 The use of dns to define stress producing events for a turbulent channel flow over a smooth wall. Flow Turb. Combust. 66, 495-512.

Nagakawa, S. \& Hanratty, T. J. 2001 Particle image velocimetry measurements of flow over a wavy wall. Physica 13, 3504-2507.

Nagakawa, S., Na, Y. \& Hanratty, T. J. 2003 Influence of a wavy boundary on turbulence. Part 1. Highly rough surface. Experientia 5, 422-436.

Offen, G. R. \& KLINE, S. J. 1975 A proposed model of the bursting process in turbulent boundary layer. J. Fluid Mech. 70, 209-228.

Panton, R. L. 1997 Self-Sustaining Mechanisms of Wall Turbulence. Computational Mechanics, Southampton.

Perry, A. E. \& Chong, M. S. 1982 On the mechanism of wall turbulence. J. Fluid Mech. 119, $173-217$.

Perry, A. E. \& Marusic, I. 1995 A wall-wake model for the turbulence structure of boundary layers. Part 1. Extension of the attached eddy hypothesis. J. Fluid Mech. 298, 361-388.

Prandtl, L. 1904 Ueber flussigkeitsbewegung bei sehr kleiner reibung. In Proc. 3rd Intl Congr. Maths, pp. 484-491. Heidelberg, Germany.

Raffel, M., Willert, C. \& Kompenhans, J. 1998 Particle Image Velocimetry - A Practical Guide. Springer. 
Robinson, S. K. 1991 Coherent motions in turbulent boundary layers. Annu. Rev. Fluid Mech. 23, 601-639.

Smith, C. R., Walker, J. D. A., Haidari, A. H. \& Soburn, U. 1991 On the dynamics of near-wall turbulence. Phil. Trans. R. Soc. Lond. A 336, 131-175.

SpalaRT, P. R. 1988 Direct simulation of turbulent boundary layer up to $R e_{\theta}=1410$. J. Fluid Mech. 187, 61-98.

TARDU, S. 1995 Characteristics of single and clusters of bursting events in the inner layer, Part 1: Vita events. Exps. Fluids 20, 112-124.

Theodorsen, T. 1952 Mechanism of turbulence. In Proc. Second Midwestern Conf. on Fluid Mech. Ohio State University, Columbus, Ohio.

Tomkins, C. D. \& Adrian, R. J. 2003 Spanwise structure and scale growth in turbulent boundary layers. J. Fluid Mech. 490, 37-74.

Townsend, A. A. 1976 The Structure of Turbulent Shear Flow. Cambridge University Press.

TRitTon, D. J. 1967 Some new correlation measurements in a turbulent boundary layer. J. Fluid Mech. 28, 439-462.

Wei, T. \& Willmarth, W. W. 1989 Reynolds-number effects on the structure of a turbulent channel flow. J. Fluid Mech. 204, 57-95.

Willmarth, W. W. \& Lu, S. S. 1972 Structure of the Reynolds stress near the wall. J. Fluid Mech. $55,65-92$.

Zhou, J., Adrian, R. J., Balachandar, S. \& Kendall, T. M. 1999 Mechanisms for generating coherent packets of hairpin vortices in channel flow. J. Fluid Mech. 387, 353-396. 


\section{University Library}

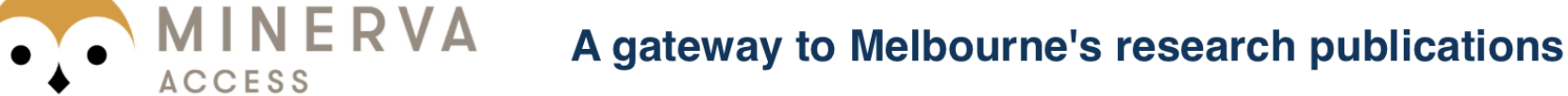

Minerva Access is the Institutional Repository of The University of Melbourne

\section{Author/s:}

Ganapathisubramani, B.;Hutchins, N.;Hambleton, W. T.;Longmire, E. K.;Marusic, I.

Title:

Investigation of large-scale coherence in a turbulent boundary layer using two-point correlations

Date:

2005

\section{Citation:}

Ganapathisubramani, B., Hutchins, N., Hambleton, W. T., Longmire, E. K., \& Marusic, I. (2005). Investigation of large-scale coherence in a turbulent boundary layer using two-point correlations. Journal of Fluid Mechanics, 524, 57-80.

Publication Status:

Published

Persistent Link:

http://hdl.handle.net/11343/34779 